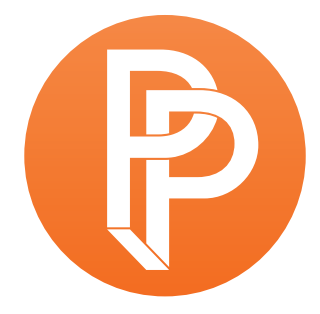

PERFORMANCE

PHILOSOPHY

\title{
NIETZSCHES DIONYSOS
}

\section{DIETER MERSCH ZÜRICHER HOCHSCHULE DER KÜNSTE}

In seinen Überlegungen zu einer ,Ästhetik des Erscheinens', betitelt mit Dionysos, hat Karl Heinz Bohrer die These vertreten, dass Nietzsches Figuration des Dionysischen einer Ästhetik des InErscheinung-tretens zuspielt, ja dass Dionysos überhaupt den Gott des Erscheinens darstellt. Zwei weitere Thesen hat er damit verbunden: Erstens, die Tragödien-Schrift Nietzsches, in der die Opposition des Dionysischen und Apollinischen als entgegengesetzte Kunstkräfte eingeführt wird, sei in erster Linie nicht als eine Theorie des Ästhetischen gedacht gewesen, sondern als "Lebenslehre", deren Kern, wie es heißt, "die elementare, materialistische Feier des Lebensimpulses und [die] Untergrabung idealistischer Vorannahmen wie Vernunft, Substanz, Subjekt" sei (Bohrer 2013,13). Zweitens, so Bohrer weiter, handle es sich, wo diese Lebenslehre ins Ästhetische gewendet werde, vor allem um eine "Ästhetik des Erhabenen“, wobei nirgends klar werde, ob sie "rezeptionstheoretisch oder produktionsästhetisch" zu verstehen sei (Bohrer 2013, 15). Ich möchte dem nicht widersprechen, zumindest nicht ganz, vielmehr eine Verschiebung oder Umakzentuierung der Grundmotive vornehmen, wobei daran zu erinnern wäre, dass Nietzsches Dionysos zwar eine direkte Provokation und einen Angriff auf die seit Winckelmann gültige KlassikDeutung darstellt, die das Apollinische zu ihrem Zentrum erhoben hat, und gegen dessen Prinzip die Einführung eines anderen, entgegengesetzten Prinzips keine genuine Erfindung Nietzsches darstellt, vielmehr der schmalen Kluft zwischen Hegel und Hölderlin ersteigt. Weist nämlich die Hegelsche Ästhetik von Anfang an auf den Schein und die Erscheinung_als notwendige Bedingung der Wahrheit, denn die Wahrheit wäre nicht, wie Hegel schreibt, wenn sie nicht schiene und erschiene-, so bleiben Schein und Erscheinung doch überall ans Kriterium des Absoluten 
gebunden, ja, die Unwahrheit des Ästhetischen liege geradezu darin, dass es gar nicht anders könne, als sich der Sprache der Erscheinungen zu bedienen. Für Hölderlin avanciert hingegen das Dionysische zu einem metapoetischen Symbol, das inn, den Schillernden und sich stets Verwandelnden, mit der Praxis der Kunst selbst verbindet.

Nietzsche knüpft hieran an, auch wenn er der Metapher eine ganz andere Wendung erteilt. Denn will man den Gegensatz bzw. die Verschiebung, die ich zu markieren versuche, auf eine Formel bringen, so müsste man sagen, dass Bohrer Nietzsche romantisch oder genauer: als Höhepunkt und Gipfel der Romantik versteht, zu der die Ästhetik des Erhabenen und die „Feier des Lebensimpulses", vor allem aber die Kritik des Idealismus, die Subversion der Auszeichnung des Rationalen gehört. Dennoch trägt Nietzsche in den Kontext der Begriffe eine Reihe charakteristischer Konversionen ein, die seine Kunsttheorie ins vollends Antiromantische rücken. Berührt ist damit die Frage, ,was' Nietzsche mit Dionysos meint-wer ,sein' Dionysos ist-und inwieweit sich Kunst in ihm, in seiner Gestalt_im Unterschied zum "schönen Schein"—eigentlich erst entfaltet. Dabei wird sich zeigen, dass der Schlüssel des mit dieser Figur verbundenen Umsturzes in der Trennung zwischen einem ganzen Arsenal von Begriffen, die exakt die traditionelle Theorie der Kunst konstituierten und die um die Metapher des Traums, der Imagination kreisten, und ihrer Auflösung, ihrer Negation liegt, die mit einer ganz anderen Metaphorizität, nämlich der Gewalt, der Destruktion und dem, was man ,Differenzsetzung' nennen könnte, assoziiert ist. Und erweist sich die erstere Vorstellung an eine Serie von Gestaltungs- und Verfahrensweisen geknüpft, genügt letztere vor allem der Figur der Zäsur, der Ent-Staltung oder des Widerstandes, woran das Ästhetische zugleich sein Reflexionsprinzip findet. Nietzsche führt dies kaum aus, vielmehr deutet er es lediglich auf indirekte Weise an. Seine Kunstphilosophie findet ihren antiromantischen Zug darin, dass sie nicht etwas, was lange vorher angelegt war, auf ihren Höhepunkt bringt, sondern, wie ich zeigen möchte, vorweist auf ein Künftiges, Anderes, dem die Notwendigkeit eines Traditionsbruchs innewohnt.

Bekanntlich erscheint das Paar Apollon/Dionysos bei Nietzsche prominent in dessen Richard Wagner gewidmeten Schrift Die Geburt der Tragödie von 1871. Etwa zeitgleich entsteht die Schrift Die dionysische Weltanschauung von 1870, die das Problem der ästhetischen Darstellung, wie die Ästhetik Hegels sie in den Mittelpunkt ihrer Philosophie der Kunst stellte und die die romantische Kunst bis an den Rand der Darstellung des Undarstellbaren trieb und ,über-trieb'-man denke vor allem an die Malereien Henry Füsslis und William Turners-im Rückgang auf antike Ansätze, vor allem der Frage nach der Mimesis, neu stellt. Zwar thematisierte Nietzsche das Mimesis-Problem nicht auf explizite Weise, wohl aber maskiert aus der Opposition zwischen dem Apollinischen und Dionysischen. Beide Begriffe, ihre Komplementarität wie ihr fortwährendes Spiel, treten damit an die Stelle dessen, was sich produktionsästhetisch als der eigentliche Kern des künstlerischen Prozesses beschreiben lässt: Die Hervorbringung von etwas, seine Darstellung im Wortsinne des Dahin- bzw. Vor-Augen-stellens. Nach Nietzsche gehören dazu indessen als "Doppelquell“ oder "Stilgegensatz" (Nietzsche 1999, KSA 1, 553, 582) die beiden „ineinander gewobenen“ und „in ihren 
höchsten Zielen verschiedenen“ "Kunsttriebe“ (Nietzsche 1999, KSA 1, 25, 38, 82, 103) des Dionysischen und Apollinischen, deren "Leitmotivik" trotz mehrfacher Umdeutungen sich durch das gesamte philosophische Werk Nietzsches hält. Noch die Götzen-Dämmerung handelt von ihr (z. B. Nietzsche 1999, KSA 6, 117-118.), genauso wie die zahllosen Stellen der Nachlass-Schriften, besonders aus der Mitte der 1880er Jahre und dem Umkreis des sogenannten Willens zur Macht, wobei eine zunehmende Radikalisierung auffällt. Der Ausdruck "Duplizität" fällt gleich zu Beginn der Geburt der Tragödie und nennt dabei noch Unentschiedenes-ich komme noch darauf zurück; entscheidend ist jedoch, dass die ästhetische Darstellung nicht in Idee und Erscheinung, wie es seit Hegel nahe liegt, zerfällt, sondern-ich adaptiere damit eine Heideggersche Formulierungeinem ,Streit' entspringt, einem Polemos oder einer Polemik, welche in einem wesentlichen Maße zum einen die Form betrifft, zum anderen aber einem Exzess angehört, der vordergründig den „Rausch" oder-im Sinne Platons-die "Mania', den schöpferischen Wahnsinn meint und im Besonderen die Besessenheit des Genies adressiert, tieferliegend, wie ich herausarbeiten möchte, die Ekstasis, ein Wort, das viele Bezüge eröffnet, vom Herausstehenden der ,Ex-sistenz'-es ist dasselbe Wort-über das Angehende der Materialität bis zu dem, was man in einem noch sehr abstrakten Sinne ,das Ereignis' nennen könnte.

Ich gehe dazu noch einmal auf den Tragödien-Text zurück, um die Hauptmerkmale zu entschälen. Einerseits heißt es dort, sei das Kunstwerk "ebenso dionysisch [...] als apollinisch [...]", wobei Nietzsche vom "gemeinsame[n] Ziel beider Triebe [...]" (Nietzsche 1999, KSA 1, 42) oder disparaten „Wege[n] zum Kunstschaffen“ (Nietzsche 1999, KSA 1, 564) spricht, sodass man den Eindruck gewinnt, als handle es sich um eine Alternative-zwei grundsätzlich verschiedene künstlerische Verfahrensweisen, die unterschiedliche Werkarten hervorbringen. So wird, wörtlich, ihr "Gegensatz" (Nietzsche 1999, KSA 1, 30) beschworen, ihre im „tiefsten Wesen und ihren höchsten Zielen verschiedenen" Positionen (Nietzsche 1999, KSA 1, 103). Andererseits betont Nietzsche noch in den 1880er Jahren, dass allererst beide Elemente zusammenkommen müssen, um Kunst überhaupt hervorzubringen, wobei unklar bleibt, auf welche Weise ihr Zusammenkommen erfolgt. Nun verdankt sich die Opposition zwischen den beiden Kräften-die als Kräfte oder Triebe ja nie rein vorkommen, sondern Energien stiften, aus denen etwas allererst hervorgehen kann-; dieser Gegensatz verdankt sich einer Anzahl begrifflicher Unterscheidungen, die dem Apollinischen wie dem Dionysischen spezifische Attribute zuschreiben. In Bezug auf Apollon ist da von der Täuschung-dem alten Mimesis-Problem, wie es von Platon diskutiert wird, ferner vom „Traumbild“ (Nietzsche 1999, KSA 1, 26f.)—der klassischen Metapher des Phantasmas, des Imaginariums-die Rede, oder auch von der "Maske" (Nietzsche 1999, KSA 1, 65), während dem Dionysischen die Kennzeichen einer "ekstatischen“ Feier sowie des "Uebermaass[es]" (Nietzsche 1999, KSA 1, 40, 565) zukommen. Sie werden in späteren Jahren eindeutiger aufeinander bezogen und zu Unterarten derselben Exzentrik erklärt: „Was bedeutet aber der von mir in die Ästhetik eingeführte Gegensatzbegriff apollinisch und dionysisch, beide als Arten des Rausches begriffen?", fragt Nietzsche etwa in der Götzen-Dämmerung (Nietzsche 1999, KSA 6, 117), wobei die Antwort auf die Frage keinen Zweifel daran lässt, dass die Ent- oder Verrückung, die dionysische Ekstase den primären „ästhetischen Grundzustand“ ausmacht, während das Imaginäre nur deren Korrelat bildet, das die Kunsthaftigkeit der Kunst allererst definiert. 
Was jedoch das apollinische und dionysische Prinzip im eigentlichen Sinne meint, kann erst deutlich gemacht werden, wenn man die Attribute beider nebeneinander stellt. Apollons Telos, heißt es z. B. in der kleineren Schrift Die dionysische Weltanschauung, sei die Gestalt, das Bild, die Statue (Nietzsche 1999, KSA 1, 563), deren Gestaltung stets dem "schönen Schein“ (Nietzsche 1999, KSA 1, 26) und ihrem "Gesetz" (Nietzsche 1999, KSA 1, 40) der "maassvolle[n] Begrenzung" (Nietzsche 1999, KSA 1, 28) gehorche, wie Nietzsche in der Geburt der Tragödie fortsetzt. Demgegenüber beschreibt Nietzsche die dionysische Kunst bzw. Kunstenergie, die er zunächst noch ganz schopenhauerisch von der "unbildlichen Kunst der Musik" (Nietzsche 1999, KSA 1, 25 , auch 34, 43ff., 103) her ableitet, aus dem "Spiel mit dem Rausche“ (Nietzsche 1999, KSA 1, 554, 555, 567, ebenso 583 u.ö.). Doch was heißt „Rausch“_im übrigen das klassische Attribut des Dionysos im Sinne des Bakchos? Der Rausch bedeutet eine Exzentizität, ein Verlassen des Zirkels dessen, was mit Schopenhauer das "principium individuationis" genannt werden kann-das Unterscheidenkönnen mitsamt seinen Verkörperungen der Repräsentation (Nietzsche 1999, KSA 1, 554, 555), worin die Dinge gleichsam an ihrem Platz und die Bäume Bäume, die Häuser Häuser und die Menschen Subjekte sind, die ihre Entscheidungen autonom und selbstverantwortlich treffen. Dagegen offenbare der "Rausch" die rohe "Gewalt der Natur" (Nietzsche 1999, KSA 1, 555). Weit über Schopenhauer hinaus, der der Musik immerhin einen Sonderstatus einräumte, sofern sie nichts darstelle, nichts repräsentiere, sondern den "Willen“ selbst manifestiere, belegt Nietzsche die Erfahrung des Dionysischen mit der Erfahrung des Chaos, worin keine Unterscheidung mehr Gültigkeit besitzt und die Dinge indifferent gegeneinander verschwimmen. Deshalb spricht Nietzsche angesichts des Dionysischen vom „Barbarischen“ (Nietzsche 1999, KSA 1, 40, auch 71), dem "Grausen“ (Nietzsche 1999, KSA 1, 28) und von dem "Schrecken“ (Nietzsche 1999, KSA 1, 39)—wobei sich hinzufügen lässt, dass der "Schrecken“-der "Sturz in die Finsternis", wie es bei Platon heißt, ebenso wie das Aristotelische "Sichverwundern" zu den ,ur-sprünglichen" philosophischen Gefühlen gehört, d. h. zu jenen Emotionen, die uns allererst zu philosophieren lehren. Was bewirken die Verwunderung, das Erschrecken? Gewiss kann man letzteres über die Traditionslinien des Pseudo-Longinus und Edmund Burke mit der Erfahrung des Erhabenen verbinden-aber zunächst erzeugt das Erschrecken einen Riss, eine Umwendung oder Katastrophē. Auch Nietzsche spricht von der "Zerreissung" (Nietzsche 1999, KSA 1, 33) aller Bindungen und Orientierungen sowie der "Vernichtung der gewöhnlichen Schranken und Grenzen des Daseins" (Nietzsche 1999, KSA 1, 56). Aber das Besondere an Nietzsche ist, dass diese Zerreißung nicht das Entsetzliche ist, das um jeden Preis vermieden werden muss, die Zerstörung der Ordnung als Lebensbedingung, sondern im Wortsinne das Ent-Setzliche, dasjenige, was uns an einen anderen Platz ,ver-setzt" und gerade dadurch ein "Nie-empfundenes" eröffnet. Kurz: Die Negativität des Risses ist überhaupt erst Bedingung eines Anderen, eines Neuen. Das Entscheidende am Dionysischen ist folglich die rückhaltlose Negation (Nietzsche 1999, KSA 1, 577), wodurch, wie es in der Tragödienschrift heißt, der "Satz vom Grunde [...] eine Ausnahme zu erleiden" und der Mensch "plötzlich an den Erkenntnissformen der Erscheinung irre" (Nietzsche 1999, KSA 1, 28) werde, aber gerade dadurch in die Nähe der "Wahrheit" der Natur und des "Lebens“ gerate (vgl. Nietzsche 1999, KSA 1, 56): „Apollo steht vor mir, als der verklärende Genius des principii individuationis, durch den allein die Erlösung im Scheine wahrhaft zu erlangen ist: während unter dem mystischen Jubelruf des Dionysus der Bann der Individuation zersprengt wird 
und der Weg zu den Müttern des Sein's, zu dem innersten Kern der Dinge offen liegt.“ (Nietzsche 1999, KSA 1, 103)

Man muss das Pathos der Formulierungen ein wenig ausstreichen, um zum Kern des Gemeinten zu gelangen. Denn repräsentiert Apollon die Sprache der Form, deren überliefertes Prinzip die Identität oder deren romantische Kritik das Fragment ist, dessen Unabschließbarkeit gleichwohl an ihrem Grundsatz festhält, weil das Siegel des "Maass[es]“ (Nietzsche 1999, KSA 1, 40) selbst noch dort gilt, wo nur mehr Brüchiges erscheint, bedeutet Dionysos die Sprache der Differenz, die in der Negation gründet und sich einzig im Negativen ausbuchstabieren lässt. Deswegen verbindet eine Nachlaß-Notiz aus dem Jahre 1885 die Gottheit mit "Diabolus“ (Nietzsche 1999, KSA 11, 473): Das singulär dastehende Wort meint weniger die Verführung, das Prinzip des Diabolischen als Negativum schlechthin, vielmehr muss es aus dem alten Gegensatz zwischen symbolon und diabolon, dem Zusammenwerfen und dem Durcheinanderwerfen, Ordnung und Chaos als miteinander korrespondierende Momente eines Spiels gelesen werden.

3.

Insbesondere erscheint hier die Negativität des Dionysischen als notwendiges Moment von Kreativität. Nietzsche denkt das Schöpferische weniger aus der creatio, als vielmehr aus dem Riss, der Differenz. Deswegen spreche ich vom Übergang einer Ästhetik der Repräsentation oder Ästhetik der Form zu einer Ästhetik der Differenz, wie er charakteristisch für den Übergang von der klassischen Kunst zur Moderne, insbesondere zu Beginn des 20. Jahrhunderts für die Avantgarden ist. Man könnte sagen: Nietzsche ahnt den Avantgardismus in der emphatischen Sprache des späten 19. Jahrhunderts vor. Was zudem pathetisch in Begriffen einer höheren Wahrheit, die später selbst noch als Illusion entlarvt wird, beschworen wird und einen weiteren Gegensatz, der das Denken Nietzsches durchzieht, anklingen lässt, nämlich den zwischen "Reflectiren" und "wahrer Erkenntniss" (Nietzsche 1999, KSA 1, 57) oder Analyse, Methode und Bestimmung auf der einen und Offenbarung auf der anderen Seite, weist auf eine elementare "Differenzerfahrung", die sich auch als das „Aufscheinen“ von „Ex-sistenz" apostrophieren lässt (vgl. Lyotard 1994 sowie Mersch 2004). Anders gewendet: Das Dionysische meint den Moment jenes Risses, der im Wortsinne dem "Aufriss" von Präsenz gleichkommt, jener grundlegenden Erschütterung, um mit Heidegger zu sprechen, „daß Seiendes ist und nicht vielmehr nicht ist" (vgl. z. B. Heidegger 1984, 2).

In immer neuen Wendungen und Formulierungen hat Nietzsche diesen außerordentlichen Moment zu fassen versucht und mystifiziert. Ich zitiere: "Jetzt öffnet sich uns gleichsam der olympische Zauberberg und zeigt uns seine Wurzeln“ (Nietzsche 1999, KSA 1, 35). Gleichzeitig spricht er von der "Erlösung" in "mystische[r] Einheitserfahrung" (Nietzsche 1999, KSA 1, 30), vom "Wahrhaft-Seienden und Ur-Einen“ und dem "wahren Blick in das Wesen der Dinge" (Nietzsche 1999, KSA 1, 56), der der "entzückenden Vision“ einer Entrückung bedarf (Nietzsche 1999, KSA 1, 38). Nietzsche scheint förmlich von seinen Formulierungen hingerissen, mitgerissen zu werden, doch haben wir es im Medium der Sprache selbst mit einem Taumel, einer Vergeblichkeit zu tun, die weniger die Disparität von Energien, auch nicht die Disparität zwischen einer Formästhetik und 
einer Ereignisästhetik heraufzubeschwören sucht, sondern eine historische Disparität anzeigt: Gegensatz zwischen dem Erbe der Tradition und dem noch Unausdrückbaren, Künftigen, das ankündigt, was erst die avantgardistische Moderne aufnehmen sollte: eine anhaltende Praktik der "Destruktion“ oder "Dekonstruktion“, die das Positive der Form, des Mediums und der Darstellung, mithin die Elemente der klassischen Ästhetik voraussetzt, um mit innen zu brechen und in sie den Differenzpunkt ihrer Auflösung einzutragen. Gleichzeitig werden ihnen zwei entgegengesetzte Erkenntnisformen zugeordnet: das Gesetz der Selbstbeschränkung und Selbsterkenntnis, die den/die Künstler_in als Autor_in und Subjekt seiner/ihrer Werke entwirft, die den Autoritäten seiner/ihrer Intentio, seiner/ihrer Inspirationen und seines/ihres Willens zum Ausdruck folgt, sowie die Erfahrung eines Wundmals, einer Verletzung an der Zeit und ihrem buchstäblich Unheilen, die den Künstler oder die Künstlerin zur Ausnahmeerscheinung, zum/zur Stigmatisierten und Ausgestoßenen stilisiert, der/die-gemäß den Selbstbeschreibungen Arthur Rimbauds, Lautréamonts oder auch Antonin Artauds-versehen mit den Insignien seines Opfers und seines Wahnsinns gleichwohl den Namen einer höheren "Wahrheit" auszusprechen vermag. Scheint Nietzsche, zumindest zur Zeit der Abfassung der Geburt der Tragödie, den Radikalisierungen der Spätromantik noch erlegen zu sein und deren innere Linie noch fortzuschreiben, wurzelt, so meine These, in der Gegenüberstellung zwischen dem Apollinischen und dem Dionysischen bereits eine tiefere Opposition, die auf den Umbruch der Epoche, ihre unausweichliche Zäsur vor-weist, die gleichzeitig die künstlerische Praktik in ein anderes Terrain überführen wird. Nietzsche deutet diese Möglichkeit nur an, ohne sie auszuführen. Sein Verweis auf die dionysische Kraft der Negation lockert so jene extreme romantische Fixierung auf das Künstlersubjekt und sein außerordentliches Genie, das Nietzsche zweifellos selbst immer verklärt hat; doch verwirft er gleichzeitig die „bisherigen“ Ausdrucksmedien, um das freizusetzen, was keine Darstellung besitzt und keine Symbolisierung duldet, denn das Andere, das Außerordentliche, Noch-Nicht-gedachte "gibt es" nur dort im Sinne eines Gebens, einer Gabe, wo die Sprache, das Bild und entsprechend die Formen der Repräsentation zerstört werden, wo mithin die "Differenz" das Mediale teilt, um durch es hindurch eine Heterogenität, ein ebenso Unsichtbares wie Undarstellbares freizulegen.

Die Unterscheidung, die auf diese Weise virulent wird, verläuft entsprechend zwischen dem Schein und der Erscheinung in der Bedeutung des "Was", das seine Geltung und Bestimmung aus der Individuation bezieht, und der "Erscheinung der Erscheinung" im Sinne des „Daß" (quod), jener Ereignishaftigkeit einer Präsenz, die nie positiv "erscheint", sondern allein nur negativ gefasst werden kann (vgl. Mersch 2002, 355ff.). Das meint auch: Solange es die Kunst mit der Form, der Darstellung oder der technē zu tun hat, bleibt sie an Medien gebunden und verfährt apollinisch; sobald sie aber diese umstürzt und überschreitet, kann das auftauchen, wofür der Begriff fehlt und die Bestimmung versagt. Das Dionysische meint nichts anderes: Das Medium konstituiert, gestaltet und versinnlicht; seine Fraktur oder Brechung konfrontiert hingegen mit einer Lücke, einem "Durchriß", wodurch ein „Unfügliches“ im Sinne eines „Aus sich“-Heraustretenden, Sich-Zeigenden zum Vorschein gelangt. Wir haben es dann mit einer "anderen“ Gegenwart zu tun, nicht einer, deren Präsenz bereits im Zeichen oder seiner Auszeichnung, seiner Rahmung oder Inszenierung verbürgt ist und die Jacques Derrida unter das Signum der "Nachträglichkeit" und der unvermeidlichen Apräsenz gestellt hat (Derrida 1972, bes. 373ff.), sondern einer, worin sich die Erfahrungen des Negativen und der Alterität kreuzen und die allein dort statthat, wo sich ein 
Widerspruch, eine Aporie ereignet. Deswegen spricht Nietzsche von der "Sprengung" des principium individuationis sowie-in missverständlicher Festhaltung an subjektphilosophischen Termini-von der "Steigerung des Subjectiven zu völliger Selbstvergessenheit" (Nietzsche 1999, KSA 1, 29), dem, wie es später heißt, "Ausser-sich-sein“ der „Ekstasis“ (Nietzsche 1999, KSA 7, 10), das einzig jenseits des Medialen, aber doch durch ein Mediales hindurch und seine Medialität ebenso unterlaufend wie zersetzend, erscheinen kann: Die Ästhetik der Differenz supponiert die Formästhetik ebenso sehr wie sie sie zerschlägt. Von einer "Steigerung des Subjectiven zu völliger Selbstvergessenheit" kann deshalb nur gesprochen werden, wo die Subjektivität des Subjekts ebenso wie die Medialität des Mediums gleichermaßen gerettet wie erschüttert und transzendiert werden. Der Übergang von der Form- zu dem, was ich Differenzästhetik nenne, impliziert daher die Entsubjektivierung der Kreativität- „das Subjektive verschwindet ganz vor der hervorbrechenden Gewalt des Generell-Menschlichen, ja des Allgemein-Natürlichen“ (Nietzsche 1999, KSA 1, 554-555), wie es in der Dionysischen Weltanschauung heißt: „Die Kunstgewalt der Natur, nicht mehr die eines Menschen, offenbart sich hier [...]." (Nietzsche 1999, KSA 1, 555) Nietzsche greift damit ebenso sehr auf jenes Diktum vom "Tod des Autors" vor, das erst später durch die Theorien des Poststrukturalismus und der Intertextualität seine eigentliche Erfüllung fand. Gleichzeitig hält er sich aber in einer systematischen Zweideutigkeit oder Unbestimmtheit, weil das Subjekt nur aufgrund der Subjektivität des „Lebens" und der Künstler bzw. die Künstlerin lediglich als ein/e im Rausch entmächtigte/r Künstler_in überwunden und "geheiligt" werden kann. Im Prinzip des Dionysischen zeichnet sich so ein Vorschein ab, etwas, auf das Nietzsche zudachte, für das die Zeit und ihre Ausdrucksmöglichkeit aber noch nicht reif war. Es fragt sich, was, wo das Subjekt fehlt, an seine Stelle treten kann-wie ebenso, was Kunst und künstlerische Verfahren bedeuten können, wo das Mediale durch seine Fraktur, seinen Riss hindurchgegangen ist.

4.

Überall steuert Nietzsche damit auf etwas zu, was ihm im selben Augenblick wieder zu entgleiten droht; erst die spätere Radikalisierung wird die Ambiguitäten zwischen Apollinischem und Dionysischem als Kunstformen oder ästhetische Prinzipien lösen. „[I]ch hatte [...] das wundervolle Phänomen des Dionysischen als der Erste begriffen“, heißt es im Ecce homo (Nietzsche 1999, KSA 6, 311); denn in seinem Erstlingswerk habe er in aller Heimlichkeit und Einsamkeit ein "Opfer" dargeboten, „ich fand Keinen, der es verstanden hätte, was ich damals that", fügt er in Jenseits von Gut und Böse hinzu (Nietzsche 1999, KSA 5, 238). Nietzsche hat insofern die Geburt der Tragödie selber als "romantisch“ verworfen—nicht nur in der später hinzugefügten "Selbstkritik", die vor allem den "Sprachkitsch“ geißelt, vielmehr findet sich unter den Aufzeichnungen zwischen 1885 und 1886 unter dem Stichwort „Zur ,Geburt der Tragödie“" die Bemerkung: „Ein Buch mit einer Artisten-Metaphysik im Hintergrunde. Zugleich ein Romantiker-Bekenntniß" (Nietzsche 1999, KSA 12, 115). Es habe die Erlösung von Illusion und Schein als die klassischen Kunstziele durch die Gewalt des Werdens gesucht, wobei die "Vernichtung auch des schönsten Scheins" den Gipfel des "dionysische[n] Glück[s]" bedeute (Nietzsche 1999, KSA 12, 116). Entsprechend wird hier ein Gegensatz zwischen klassischer und romantischer Kunst einerseits und dionysischer (Nietzsche 1999, KSA 12, 117) andererseits konstruiert, letztere versehen mit dem Flor einer ebenso 
zerstörenden wie gebärenden, einer gleichermaßen schaffenden wie vernichtenden Praxis, die die überlieferte Ästhetik der Darstellung und Gestaltung hinter sich lässt. Was tritt an ihre Stelle? Was heißt: Differenz-Ästhetik?

Ich versuche tastend eine Annäherung. Nietzsche entzieht erstens der Kunst den/die Künstler_in und denkt so auf ein Kunstverständnis zu, das den souveränen „Formwillen“—Prinzip aller Künste bis zur Romantik—sowenig erfordert wie vorgreifende Inspirationen: „Das Kunstwerk, wo es ohne Künstler erscheint, z. B. als Leib [...]", lautet ein Fragment des Nachlasses: „Inwiefern der Künstler nur eine Vorstufe ist. Was bedeutet das ,Subjekt'? - " (Nietzsche 1999, KSA 12, 118-119) Beide Bestimmungen gehören zusammen: das Dionysische als das Negative-und das Dionysische als Desubjektivierung, als Entzug der Souveränität. Die zitierten Notizen fallen etwa zeitgleich zusammen mit der Zuspitzung des Rausches als ästhetisches Prinzip in der Götzen-Dämmerung. Hatte Nietzsche in der Dionysischen Weltanschauung z. B. noch vom "Spiel mit dem Rausch" gesprochen, heißt es nunmehr: „Damit es Kunst gibt, damit es irgend ein ästhetisches Tun und Schauen gibt, dazu ist eine physiologische Vorbedingung unumgänglich: der Rausch“ (Nietzsche 1999, KSA 6, 116). Und als das Wesentliche am Rausch wird die Entgrenzung, die Negation des Willens gesehen, der umgekehrt eine "Kraftsteigerung" (Nietzsche 1999, KSA 6, 116) entspricht, man könnte ergänzen, eine "Kraft" im Sinne der „Überfülle des Lebens". Entsprechend ist die gesamte spätere Philosophie Nietzsches überhaupt durch eine Ausweitung des dionysischen Prinzips gekennzeichnet: Heidegger hat in seiner Nietzsche-Interpretation daran angeknüpft und die "Fülle“ und "Kraftsteigerung" als „Wille zur Macht" und die Kunst als dessen "ausgezeichnete Gestalt" gedeutet (Heidegger 1985, 108-109), die das genaue "Gegenteil" von Kants „interesselosem Wohlgefallen“ bezeichnet, und zwar in doppelter Weise, nämlich einmal in Ansehung des ästhetischen Urteils, das die Kunsterfahrung an die Rezeptivität bindet, zum anderen hinsichtlich der Passivität der Aufnahme und der „Freigabe" an das, "was ist" (Heidegger 1985, 128-129). Erstens also Entsubjektivierung_oder besser: Entmächtigung des Subjekts. Deren Korrelat ist zweitens die Zentrierung des Ästhetischen auf den Leib. Leibapriori meint nicht: Vorrang der Intensität, des Überschusses bzw. das, was Nietzsche immer wieder mit dem Ausdruck „Kraft" belegt, sondern jene „Exzentrik“ einer außer-sich-seienden Positionalität, die wiederum Heidegger mit einem "Leiben“ eines „Leibs“ in Verbindung bringt (Heidegger 1985, 118-119). Man könnte sagen: Der Leib induziert hier eine Produktivität aus Affektion, eine nichtintentionale Dynamik, die ans Pathische einer „Besessenheit“ rührt, d. h. an die Passivität einer Alterität (Mersch 2006).

Nietzsche, wie ebenfalls Heidegger betont, fragt somit nicht nach dem Werk als einem Resultat, als Ort einer Rezeption, sondern primär nach Prozessen und deren Vollzügen, ihren Wirkungen, nach dem, was nicht eine Absicht und deren Verkörperung ist, sondern ein ästhetisches Denken gleichsam im Tun und durch das Tun bedeutet-ein Denken, das nicht der Handlung entgegengesetzt ist und sie unterbricht, sondern ihr als eigene Erkenntnisform, als nichtdiskursives und nicht diskursivierbares Wissen entspringt. Eine wesentliche Methode dafür im Wortsinne von meta hodos, einem Weg folgen, oder vielleicht besser: im Sinne eines poros, einer immer schon im Materiellen und Leiblichen zu vollziehenden Passage, also eines metaporos oder sogar diaporos, das Durchlässigkeit verlangt, ist neben der Desubjektivierung und der durch 
alle Poren-das gleiche Wort!-hindurch vollzogenen Pathik drittens das, was ich nun schon mehrfach als Bruch oder Unterbrechung, als die buchstäbliche Abgründigkeit eines Risses zu apostrophieren versucht habe. Dieser geht von der künstlerischen Produktion aus, wie er durch sie durchgeht und aus ihr heraus geschieht. Nietzsches produktionsästhetisch begründeter Zusammenhang zwischen Kunst und Ereignung fußt auf dieser Differenzsetzung. Das ästhetische Ereignis ist die Differenz, folgt ihr, wie umgekehrt die Differenz erst durch die Instaurierung eines Risses gleichsam im Innern der ästhetischen Prozesse hervorgeht. Wie ist das verständlich zu machen? Bei Nietzsche bleibt vieles noch zu unbestimmt-weil, so auch der Kommentar Heideggers, bei ihm „[a]lles [...] zur Kunst [gehört]. Die Kunst wäre dann nur ein Sammelwort, nicht der klare Name einer in sich gegründeten und ausgegrenzten Wirklichkeit" (Heidegger 1985, 142). Ersichtlich besteht das Problem darin, dass auf diese Weise zwar eine kreative Produktivität beschworen, nicht aber eigentlich verstanden werden kann; sie gleicht vielmehr dem Leben, der Präsenz des Leibes, seiner Mystifikation, die sie einem "Willen zur Macht" zuordnet und die dadurch nicht minder metaphysisch argumentiert, wie die Kunstauffassung, gegen die sie streitet-besonders die Kunstauffassungen Platons und Hegels. Gegen beide totalisiert Nietzsche die Fülle des Lebens und stilisiert ihre Entfaltung zur künstlerischen Tat. Dagegen käme es auf eine angemessene Rekonstruktion der jeweiligen Strategien artistischer Produktion an, d. h. auf jene konkreten Differenzfiguren, denen sie aufruhen, um je spezifische "Ereignisse der Differenz" allererst statuieren zu können.

5.

Lassen Sie mich dazu am Schluss ein paar weiterführende Gedanken skizzieren. Dazu nutze ich das Stichwort der ,ästhetischen Strategie', die die künstlerische Arbeit und Arbeitsweise betrifft, die auch eine Arbeit mit dem Leib bedeuten kann, aber sich nicht notwendig darin erschöpft. Weit eher möchte ich an die Vorstellung einer Konstellation von Praktiken erinnern, wie sie für die Ästhetik Adornos eine zentrale Rolle spielen, und die zunächst nichts anderes tun, als im Moment des con stellare, d. h. einer Streuung oder ,Fügung' von Momenten ihre Nichtpassung, ihre Lücken oder, wieder im Wortsinne, Verwerfungen und Ent-Stellungen preiszugeben. Dazu ist die Erfahrbarmachung eines ,Zwischen' erforderlich. Sie geschieht im Performativen kraft jener Klüfte und "Chiasmen", die dadurch ein Differenzereignis statuieren, das das Unfügliche der Fügungen, ihr Sichverweigerndes, auch die Gewalt der Synthesis noch etwas weiter geöffnet, gelockert und aufgerissen wird. Das bevorzugte Mittel dazu ist der Widerspruch, der sich einzig indirekt manifestieren lässt-in den Zwischenräumen der Szenen oder ihren Leerstellen und Abständen, z. B. im Sperrigen der Bilder, Worte und Töne, wie auch in den Störungen und Dysfunktionalitäten, worin Medien ihre Medialität enthüllen. Nietzsches „Ekstasis“ wäre hier einzusetzen-in der Auflösung der Konturen z. B. durch die konträre Verwendung von Figurationen, in Umkehrungen, und zwar so, dass die ins Spiel gebrachten Materialien ihre Materialität, die zur Anwendung kommenden Techniken ihre Ambiguität offenbaren. Anders gewendet: Die Produktion von Paradoxa nimmt für das, was ich Differenzästhetik nenne, eine ausgezeichnete Stellung ein. Paradoxa bahnen die bereits angesprochenen Wege oder Passagen, gleich Sprüngen, die nicht entworfen oder antizipiert werden können, sondern die nur erprobt oder ausgetestet werden 
können. Zugleich halten sie die Arbeit im Ästhetischen so in der Instabilität, dass Verdrängtes oder Nichtreproduzierbares zum Vorschein gelangen kann. Ich unterstreiche das ,Kann', die Möglichkeit, nicht das Muss: Die künstlerische Praxis hat an solchen-ich referiere notorisch auf die verborgene Bedeutung der Ausdrücke-,Ex-perimenten', solchen Durchgängen oder Fahrten, ihr Exerzitium, d. h. ihre reflexive Askesis. Was diese sein kann, kann weder vorausgesagt noch kanonisiert oder gelernt werden: Es entsteht mit der ganzen Kraft des Neutrums. Differenzästhetik heißt: Vervielfältigung der Klüftungen oder Spaltungen, aus deren Abständen und Kontrast im Sinne des contra-stare, der buchstäblichen Zusammen-Setzung als Gegen-Stellung ein Unfassbares, Anderes hervor-springt, ohne den Gesetzen der Kausalität zu gehorchen oder ableitbar zu sein; eine Singularität der Ereignung, die gleichzeitig etwas sichtbar, erfahrbar, erkennbar macht, was auf anderen Wegen nicht erschlossen werden kann, d. h. die ,Er-Ringung' eines außerordentlichen, anders nicht zu gewinnenden Wissens.

Die Intuition Nietzsches bleibt dafür überall richtungsweisend, vorweisend, wenn auch nur andeutend, gleich einem Wink, einer Berührung. Anders gewendet und noch einmal der Sprache Nietzsches angelehnt: Nicht die Form, die Gestalt, das ,Was' und damit das Bestimmbare, Individuierbare erscheinen maßgeblich, sondern das Nichtbestimmbare, die Destruktion, die für ein anderes Erscheinen,frei-gibt'. Ästhetische Differenzen wie auch praktische Paradoxa legen dorthin eine Spur. Sie bezeichnen keinen Selbstzweck, kein unverbindliches l'art pour l'art, an denen die Kunst ihr Lustprinzip fände, sondern sie bilden die Medien einer reflexiven Praxis, die im Singulären statthat und deren Poren und Passagen im Sinne des Dia- oder Metaporos Andeutungen oder Weisungen gleichen. Sie induzieren, in ihrer ganzen Vorläufigkeit und in ihrem experimentellen Status die Spezifik ästhetischer Episteme, die dem Praktischen selbst entstammen. Der Künstler oder die Künstlerin tritt hinter sie zurück und versteht sich dabei weniger als ein/e Schöpfer_in oder ein/e Arbeiter_in an Effekten, als vielmehr als ein Arrangeur oder eine Arrangeurin des Überraschenden und Unberechenbaren. Er/sie fungiert, mit einem Wort, nicht länger als maître de plaisir, sondern als maître de paradoxe. 


\section{Bibliographie}

Bohrer, Karl Heinz. 2013. „Dionysos. Eine Ästhetik des Erscheinens.“ In Sichtbarkeiten 1: Erscheinen, herausgegeben von Mira Fliescher, Goppelsröder Fabian, Dieter Mersch, 13-38. Zürich: diaphanes.

Derrida, Jacques. 1972. „Das Theater der Grausamkeit und die Geschlossenheit der Repräsentation.“ In Die Schrift und die Differenz, übersetzt von Rodolphe Gasché, 351-379. Frankfurt am Main: Suhrkamp.

Heidegger, Martin. 1984. Grundfragen der Philosophie. Gesamtausgabe, Bd. 45. Frankfurt am Main: Klostermann. 1985. Nietzsche: Der Wille zur Macht als Kunst. Gesamtausgabe, Bd. 43. Frankfurt am Main: Klostermann.

Lyotard, Jean-François. 1994. Analytik des Erhabenen. Kant-Lektionen. Übersetzt von Christine Pries. München: Fink. Mersch, Dieter. 2002. Was sich zeigt. Materialität, Präsenz, Ereignis. München: Fink.

_-_. 2004. „Das Entgegenkommende und das Verspätete. Zwei Weisen, das Ereignis zu denken: Derrida und Lyotard." In Im Widerstreit der Diskurse, herausgegeben von Dietmar Köveker, 69-108. Berlin: Wissenschaftsverlag.

2006. „Besessenheit. Zur Struktur des Verlangens.“ In Große Gefühle, herausgegeben von Ottmar Ette und Gertrud Lehnert, 101-114. Berlin: Kadmos.

Nietzsche, Friedrich. 1999. Sämtliche Werke. Kritische Studienausgabe in 15 Einzelbänden. Herausgegeben von Giorgio Colli und Mazzino Montinari. 3. Auflage. München/Berlin/New York: DTV Walter de Gruyter [=KSA].

\section{Biographie}

Dieter Mersch studierte Mathematik und Philosophie an der Universität zu Köln und der Ruhr-Universität Bochum. Er arbeitete von 1983 bis 1994 als Dozent für Wirtschaftsmathematik an der Universität zu Köln und an der Fachhochschule Köln. Zwischen 1983 und 1997 arbeitete er ebenfalls als freier Autor für verschiedene öffentlichrechtliche Rundfunkanstalten in Deutschland.

Er wurde 1992 mit einer Dissertation in Philosophie über die Semiotik, Rationalität und Rationalitätskritik an der Technischen Universität Darmstadt zum Dr. phil. promoviert. Danach war er bis 1997 freier Autor für verschiedene öffentlich-rechtliche Rundfunkanstalten. Von 1997 bis 2000 war er Wissenschaftlicher Mitarbeiter an der TU Darmstadt und habilitierte sich 2000 in Philosophie mit der Arbeit Materialität, Präsenz, Ereignis. Untersuchungen zu den Grenzen des Symbolischen. Von 2001 bis 2004 war er Gastprofessor für Kunstphilosophie an der Muthesius Kunsthochschule Kiel. Von 2004 bis 2013 hatte er einen Lehrstuhl für Medienwissenschaft an der Universität Potsdam inne. Seit 1. Oktober 2013 ist Dieter Mersch Leiter des Instituts für Theorie (ith) der Zürcher Hochschule der Künste.

Dieter Mersch ist Mitglied der Deutschen Gesellschaft für Philosophie, der Deutschen Gesellschaft für Ästhetik, der Gesellschaft für Musik und Ästhetik (Freiburg), der Gesellschaft für Medienwissenschaft sowie der DeutschUngarischen Gesellschaft für Philosophie. Zudem ist er Beiratsmitglied der Zeitschrift für Kulturphilosophie und Herausgeber des Internationalen Jahrbuchs für Medienphilosophie. Seine Arbeitsschwerpunkte befinden sich in den Gebieten Medien-, Kunst- und Sprachphilosophie, Philosophie des 19. und 20. Jahrhunderts sowie Semiotik und Ästhetik.

(C) 2017 Dieter Mersch 